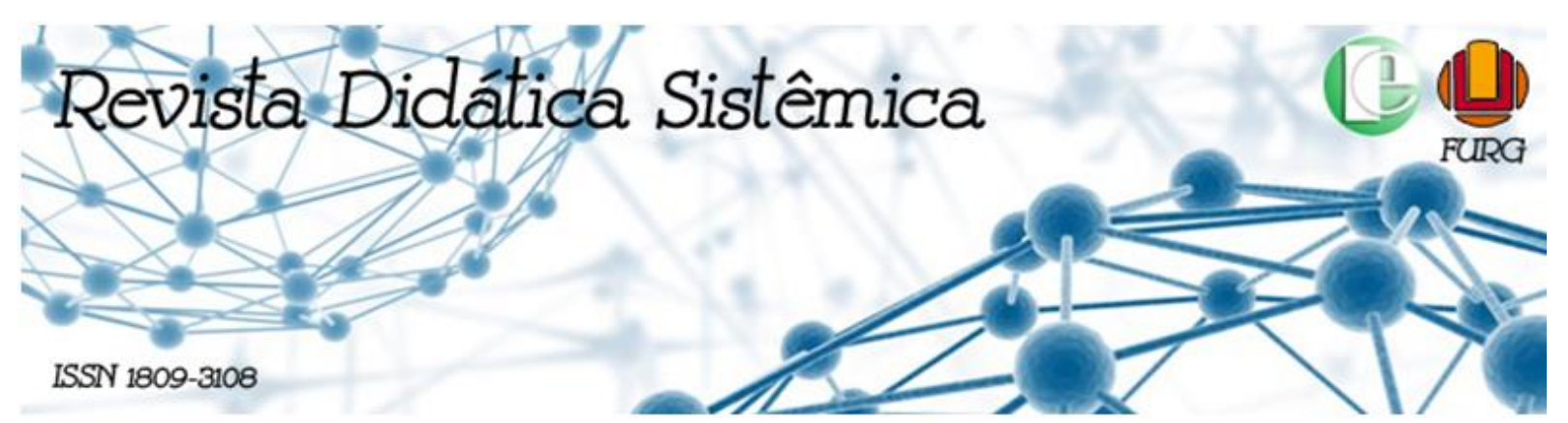

\title{
OS EFEITOS NA APTIDÃO FÍSICA DE ALUNOS DO ENSINO MÉDIO A PARTIR DE UMA UNIDADE DIDÁTICA
}

Fabiana Ritter Antunes ${ }^{1}$
Diego Colpo

\section{Resumo}

O objetivo deste trabalho é relatar os efeitos na aptidão física de alunos do ensino médio a partir da aplicação de uma unidade didática. Este estudo foi realizado com escolares de uma escola da região Centro-Oeste do estado do Rio Grande do Sul, que foram avaliados pelos testes do Projeto Esporte Brasil 2015 (PROESP). A amostra foi com um grupo de quinze alunos do ensino médio com idade entre 15 e 17 anos. Os procedimentos para a execução dos testes seguiram as orientações do PROESP 2015, sendo realizados os testes de Agilidade, Flexibilidade, Força de Membros Superiores, Inferiores e abdominais, Resistência Geral, Velocidade de Deslocamento. Os testes foram aplicados antes e após a aplicação de uma Unidade Didática com o tema capacidades física. Ao término do estudo percebesse uma melhora significativa na aptidão física dos alunos participantes da pesquisa.

Palavras-chave: Aptidão Física; Educação Física Escolar; Testes Motores

\section{THE EFFECTS ON THE PHYSICAL FITNESS OF MIDDLE SCHOOL STUDENTS FROM A TEACHING UNIT}

\begin{abstract}
This monograph aims at reporting the effects in high school students' physical fitness by means of a teaching unit. The study was conducted with students from a school located in the Center-West region of Rio Grande do Sul State. Fifteen students, aged from 15 to 17 years-old, were evaluated by 2015 Brazil Sport Project (PROESP) tests. The evaluation procedures followed 2015 PROESP orientation, that includes tests of agility, flexibility, upper and lower limbs strength, abdominal muscles strength, physical resistance, and traverse speed.
\end{abstract}

Key words: Human motricity tests - Physical education at school - Physical fitness

\section{EFECTOS SOBRE LA APTITUD FÍSICA PARA ESTUDIANTES DE SECUNDARIA DE UNA UNIDAD DIDÁCTICA}

\section{Resumen}

El objetivo de este estudio es dar a conocer los efectos sobre la condición física de los estudiantes de secundaria de la aplicación de una unidad didáctica. Este estudio se realizó con estudiantes de una región del Medio Oeste Escuela Estado de Rio Grande do Sul, que fueron evaluados por el Proyecto de prueba Sport Brasil 2015 (PROESP). La muestra con un grupo de quince estudiantes de secundaria

\footnotetext{
${ }^{1}$ Doutora da Universidade Regional do Noroeste do Estado do Rio Grande do Sul - UNIJUÍ, Brasil.

${ }^{2}$ Graduado em Educação Física-Universidade Regional do Noroeste do Estado do Rio Grande do SUL UNIJUI, Brasil.
} 
de 15 a 17 años. Los procedimientos para las pruebas siguieron la orientación PROESP 2015 y realizaron pruebas de la movilidad, la flexibilidad, la fuerza de las extremidades superiores e inferiores y abdominal, fuerza general, Speed Shift. Las pruebas se aplicaron antes y después de la aplicación de una unidad didáctica con las capacidades físicas temáticos. Al final del estudio notado una mejora significativa en la condición física de los participantes de los estudiantes de investigación.

Palabras clave: Acondicionamiento Físico; Educación Física; Pruebas de Motores

\section{INTRODUÇÃOO}

De maneira geral, é possível considerar que a evolução de um indivíduo, é viabilizada conforme as influências e ações que recebe durante o decorrer de sua vida, estando presente desde o nascimento e acompanhada até a morte. É um processo que está relacionado com a idade, mas que não depende dela.

Para a evolução do individuo as aulas de Educação Física - EDF voltadas para aptidão física atuam no processo de evolução do aluno, também tiram o mesmo da zona de conforto, saindo da rotina das aulas somente voltadas ao esporte. Os benefícios da aptidão física vão muito mais além do que somente saúde, traz benefícios para a mente e melhoras nas tarefas do cotidiano.

A Educação Física Escolar segundo González e Fensterseifer (2014, p. 264) tem como objetivo "formar indivíduos dotados de capacidade crítica em condições de agir autonomamente na esfera da cultura corporal de movimento e de forma transformadora como cidadãos políticos". Nesse sentido, a EDF atua como interlocutora desse processo de aprendizagem e construção de conhecimento, desempenhando papel importante no desenvolvimento do aluno. Tendo assim, a consciência em relação às características motoras, viabilizando conhecer as experiências que consequentemente irão garantir uma progressão dentro dos padrões. Para que os professores atinjam um desempenho considerável, é necessário um planejamento dos conteúdos que irão ser desenvolvidos em aula.

O planejamento das aulas deve ser de acordo com o qual o objetivo que o professor deseja alcançar ou transmitir para seus alunos. O planejamento deve ser realizado após o diagnóstico dos problemas do tema que vai ser trabalhado com a turma, para que assim consiga atingir os resultados esperados.

Um dos instrumentos utilizados por professores como possibilidade de planejamento dentro da Educação Física seria a Unidade Didática (UD). Esse instrumento tem como objetivo organizar, definir e especificar os conteúdos que serão trabalhados. Para Borges (2014, p. 63) "Essa divisão pode ser feita através da divisão dos conteúdos de acordo com o 
número de aula. Dito de outra, trata-se de evidenciar o conteúdo/objetivo de cada aula. Esse processo é chamado de Unidade Didática (UD)”.

Uma UD apresenta uma sequência de proposições que devem estar de acordo com as necessidades e peculiaridades do grupo com que se pretende trabalhar. Buscando conhecer as relações que se estabelecem a partir de um processo de ações, constituindo de forma significativa os possíveis efeitos do uso desse instrumento. Deve ser planejado o conteúdo agrupado com os objetivos conceituais, procedimentais e atitudinais, neste caso, a influência no desenvolvimento da aptidão física de um grupo de alunos.

Para identificar a influência e/ou melhoras na aptidão física dos alunos que participarão do estudo, será aplicado os testes de aptidão física da plataforma Projeto Esporte Brasil - PROESP 2015. Os testes motores tendem a identificar os possíveis efeitos, conforme o sistema de avaliação do PROESP: força membros superiores, inferiores e abdominal; agilidade; flexibilidade; capacidade cardiorrespiratória; velocidade de deslocamento. Sendo estes testes utilizados antes e após a aplicação da Unidade Didática, no intuito de realizar uma comparação de resultados obtidos.

Esta pesquisa tem como objetivo geral: Avaliar os efeitos da aptidão física em alunos do ensino médio da Região Centro Oeste do RS. E como objetivos específicos, podemos mencionar: Verificar a aptidão física dos alunos do ensino médio de uma Escola da Região Centro Oeste do RS; Avaliar a aptidão física antes e após os testes de força membros superiores, inferiores e abdominal, agilidade, flexibilidade, capacidade cardiorrespiratória, velocidade de deslocamento dos alunos do ensino médio de uma Escola da Região Centro Oeste do RS; Verificar se as aulas com a metodologia da utilização de circuitos sobre as capacidades físicas têm efeitos na aptidão física dos alunos do ensino médio de uma Escola da Região Centro Oeste do RS que participaram da pesquisa e ainda; Comparar as diferenças entre os sexos e idades dos alunos do ensino médio de uma Escola da Região Centro Oeste do RS.

\section{CAMINHO METODOLÓGICO}

Esta pesquisa contou com uma abordagem de cunho quali-quantitativo. No entendimento de Giddens (2012) a pesquisa é capaz de ser realizada pela forma mista, qualitativos e quantitativos de modo para conseguir uma percepção e esclarecimento mais amplo do tema estudado. 
Caracteriza-se como descritivo com ênfase na pesquisa-participante, que segundo Gil (2008, p. 31) se "caracterizam pelo envolvimento dos pesquisadores e dos pesquisados no processo de pesquisa" e ainda procura incentivar a autoconfiança a partir do preparo e uma ligação independente do exterior.

Na cidade de Jaguari - RS existem duas escolas de Ensino Médio para que esta pesquisa fosse realizada ocorreu um sorteio, sendo que a sorteada foi a "Escola 2". A cidade de Jaguari - RS está localizada na Região Centro-Oeste do Estado do Rio Grande do Sul.

Os sujeitos que aceitaram participar desse estudo, foram um total de 15 alunos, com idade entre 15 e 17 anos, sendo 6 meninas e 9 meninos, todos alunos do Ensino Médio da referida Escola. O Ensino Médio é composto por três turmas de $2^{\circ}$ ano, identificadas como $2^{\circ} \mathrm{A}, 2^{\circ} \mathrm{B}$ e $2^{\circ} \mathrm{C}$. A turma escolhida foi o $2^{\circ} \mathrm{B}$ devido ao horário das aulas de Educação Física não chocarem com o horário de trabalho do pesquisador.

Primeiramente foi realizado contato com a escola para realização da pesquisa, entregando a Carta de Apresentação. Depois de autorizada, foi agendada uma conversa com a professora de Educação Física responsável pela turma, onde foi apresentada a pesquisa, os objetivos, bem como todo o processo de desenvolvimento.

Logo em seguida foi realizada uma apresentação da proposta da pesquisa para a turma do $2^{\circ}$ ano $\mathrm{B}$, bem como, a explicação de todos os testes que seriam realizados. Os testes do PROESP(2015) foram realizados em data e local marcado com antecedência de uma semana, para definir uma primeira amostragem dos alunos. Em seguida foi desenvolvida uma Unidade Didática, com duração de 20 encontros, iniciando às 7 h e 40 min até às 9 h e 20 min, com duração de 50 minutos cada, totalizando três meses. Após as vinte intervenções os testes foram aplicados novamente, com intuito de comparação entre o inicial e o final. O primeiro teste foi aplicado no dia 09/09/16 e o (re)teste foi aplicado no dia 30/11/17.

Para a coleta das informações foram utilizados os seguintes instrumentos, uma ficha individual dos alunos para a coleta dos resultados e os testes propostos por Gaya (2015) que avaliam aptidão física. Para avaliar a flexibilidade foi utilizado o teste do Banco de Wells, já a capacidade cardiorrespiratória (VO2 Max) foi utilizada a corrida/caminhada de 6 minutos, contando com o teste do quadrado para avaliação de agilidade.

No teste de força dos membros superiores foi utilizado o medicine-ball, já o teste do salto na horizontal foi utilizado para membros inferiores. Para finalizar a bateria aplicada com a turma do Ensino Médio foi realizado teste de abdominal em 1 minuto, para verificação de força abdominal, o teste dos 20 metros para acompanhamento da velocidade de deslocamento. 
Os dados foram analisados por meio de comparação inicial e final a partir da tabela indicada pelo PROESP (2015), separando os alunos por idade e sexo. Ainda, foi realizada uma análise descritiva, com amostragem em algumas tabelas para compreensão dos resultados obtidos. A pesquisa seguiu os princípios éticos em todo o desenvolvimento da pesquisa. Todos os participantes e responsáveis assinaram o Termo de Consentimento Livre Esclarecido.

\section{RESULTADO E DISCUSSÕES}

Para identificar a influência e/ou melhoras na capacidade física dos alunos que participaram do estudo, foram aplicados os testes da plataforma Projeto Esporte Brasil 2015 (PROESP), o qual tem como objetivo avaliar os efeitos da aptidão física em alunos do ensino médio da Região Centro Oeste do Estado do RS.

Participaram do estudo quinze escolares, sendo nove do sexo masculino e seis do sexo feminino, com idade entre 15 e 17 anos. Nos primeiros testes realizados os níveis de aptidão física dos participantes estavam baixos, como por exemplo, no teste de Agilidade do sexo masculino apenas 33,3\% estavam com desempenho bom e 0,00\% do sexo feminino e após a realização da intervenção esse índice subiu para os meninos em $88,8 \%$ de desempenho bom ou superior e no sexo feminino esse numero sumiu para $66,4 \%$ desempenho bom ou superior. No teste de Velocidade de Deslocamento os primeiros resultados coletados foram de que apenas $22,2 \%$ dos meninos estavam com o desempenho bom e 33,2\% das meninas, após a intervenção esse número subiu para 100\% com o desempenho no mínimo bom ou muito bom, e 66,4\% desempenho bom ou superior. Já no teste de Resistência Geral apenas 44,4\% dos meninos estava com o desempenho bom e 16,6\% das meninas, e no segundo teste os resultados subiram para $77,7 \%$ dos meninos com desempenho no mínimo bom e 33,2\% das meninas com desempenho bom.

No teste de força dos membros inferiores os primeiros dados coletados foram de $33,3 \%$ dos meninos e 33,2\% das meninas estavam com desempenho bom e após a intervenção subiu para $66,6 \%$ dos meninos e $50 \%$ das meninas. Nos testes de membros superiores o primeiro teste teve apenas $11,1 \%$ dos meninos com desempenho bom ou acima e $0,0 \%$ das meninas, após as aulas esse numero subiu para 22,2\% nos meninos e 16,6\% nas meninas com desempenho no mínimo bom. E nos testes de força abdominal 0\% dos meninos e meninas atingiram o desempenho bom no primeiro teste, já no segundo teste esse numero subiu para $22,2 \%$ dos meninos e $50 \%$ das meninas atingiram a área sem risco a saúde. Outro teste 
aplicado foi o de flexibilidade, no primeiro teste $55,5 \%$ dos meninos e $33,2 \%$ das meninas atingiram a área sem risco a saúde, no segundo teste realizado $77,7 \%$ dos meninos e $50 \%$ das meninas atingiu a área saudável. Comparando os resultados do primeiro e segundo teste, fica evidente que a intervenção realizada pelo pesquisador trouxe resultados positivos para o nível de aptidão física dos participantes da pesquisa. Possivelmente com um tempo maior para trabalhar as capacidades físicas os resultados poderiam ser mais satisfatórios.

A tabela 1 descreve as características gerais dos participantes como Altura, Peso, Envergadura e o Índice de Massa Corporal. Podemos observar na tabela a diferença entre os resultados do sexo masculino e feminino:

\begin{tabular}{|l|l|l|l|l|l|}
\hline Meninos & Idade & Peso $(\mathbf{K g})$ & Altura $(\mathbf{c m})$ & Envergadura $(\mathbf{c m})$ & IMC \\
\hline Aluno01 & 17 & 77 & 180 & 180 & 23.8 \\
\hline Aluno02 & 16 & 64 & 173 & 173 & 21.4 \\
\hline Aluno03 & 16 & 62 & 171 & 168 & 21.2 \\
\hline Aluno04 & 15 & 60 & 177 & 177 & 19.2 \\
\hline Aluno05 & 16 & 75 & 182 & 182 & 22.6 \\
\hline Aluno06 & 15 & 68 & 180 & 177 & 21 \\
\hline Aluno07 & 15 & 74 & 176 & 175 & 23.9 \\
\hline Aluno08 & 17 & 76 & 175 & 175 & 24.8 \\
\hline Aluno09 & 15 & 67 & 181 & 181 & 20.5 \\
\hline
\end{tabular}

\begin{tabular}{|l|l|l|l|l|l|}
\hline Meninas & Idade & Peso $(\mathbf{K g})$ & Altura $(\mathbf{c m})$ & Envergadura $(\mathbf{c m})$ & IMC \\
\hline Aluno10 & 17 & 61 & 153 & 146 & 26.1 \\
\hline Aluno11 & 15 & 46 & 158 & 142 & 18.4 \\
\hline Aluno12 & 16 & 52 & 173 & 165 & 17.4 \\
\hline Aluno13 & 16 & 64 & 177 & 174 & 20.4 \\
\hline Aluno14 & 17 & 60 & 172 & 172 & 20.3 \\
\hline Aluno15 & 17 & 53 & 164 & 150 & 19.7 \\
\hline
\end{tabular}

Tabela 1: Características gerais dos participantes 
Conforme a tabela 1 a classificação dos resultados relacionados à saúde conforme o manual do PROESP (2015) verificou que $66.7 \%$ dos alunos do sexo masculino e $83.4 \%$ do sexo feminino encontram-se na zona saudável, que são valores estabelecidos por idade e sexo que não se encontram com riscos a saúde.

Segundo Pereira et al. (2012) é importante a manutenção e desenvolvimento da aptidão física, pois com o envelhecimento acarreta em perdas fisiológicas que aumentam a diminuição da aptidão física. Assim aumenta o risco de doenças regenerativas, o surgimento das doenças associadas à baixa aptidão física pode ter relação com os fatores genéticos, ambiente, e o meio.

As diferenças de gênero ocorrem devido a fatores genéticos entre meninos e meninas, Farias et al (2010) fala que algumas diferenças são devido ao acumulo de gordura entre as meninas durante a puberdade, meninas tem maior flexibilidade que meninos, vantagens anatômicas nos meninos favorecendo na função fisiológica aumentando a eficiência na produção de energia e também ao maior ganho de força associado ao hormônios produzidos na puberdade.

No teste de Agilidade conforme mostra a Tabela 2, observamos uma grande evolução dos alunos de ambos os sexos. Verificando os alunos do sexo masculino podemos perceber que $55.5 \%$ atingiram um desempenho muito bom, $33.3 \%$ bom e apenas $11.1 \%$ razoável. E no sexo feminino podemos perceber que $49.8 \%$ atingiram um desempenho bom, $16.6 \%$ muito bom e $16.6 \%$ razoável.

Para a classificação do desempenho usamos a tabela do PROESP (2015), a mesma esta dividida por gênero, idade e tempo. O desempenho pode ser excelente, muito bom, bom, razoável e fraco, verificasse o tempo em que o aluno realizou o teste e a sua idade.

Fala Bergmann et al (2015) que a ausência de práticas especifica de agilidade nas aulas de Educação Física diminui a oportunidade dos alunos de melhorarem suas capacidades coordenativas, habilidade motora, cognitiva e social. Sendo assim essa ausência pode levar o aluno não se sentir com habilidade motora para realizar tarefas pertencentes a algumas práticas corporais.

Ainda seguindo o pensamento de Bergmann et al (2015, p. 77) "embora aspectos conceituais possam contribuir para o aumento do nível de atividade física de adolescentes e consequentemente melhorar o nível de Aptidão Física”. Neste estudo, através dos dados coletados fica evidente o efeito de um trabalho de intervenção na escola com o tema 
capacidades físicas, em que as aulas elaboradas podem contribuir para o aumento dos níveis de agilidade dos alunos.

A seguir podemos observar a tabela com os resultados dos testes de agilidade que foram aplicados nos escolares com os resultados do primeiro e segundo teste, mostrando a sua evolução.

\begin{tabular}{|l|l|l|}
\hline Meninos & $\begin{array}{l}1^{\circ} \text { teste } \\
\mathrm{T}(\mathrm{s})\end{array}$ & $\begin{array}{l}2^{\circ} \text { Teste } \\
\mathrm{T}(\mathrm{s})\end{array}$ \\
\hline Aluno01 & 6.42 & 6.00 \\
\hline Aluno02 & 6.00 & 5.65 \\
\hline Aluno03 & 5.83 & 5.60 \\
\hline Aluno04 & 6.62 & 5.08 \\
\hline Aluno05 & 5.70 & 5.02 \\
\hline Aluno06 & 5.65 & 5.23 \\
\hline Aluno07 & 6.00 & 5.28 \\
\hline Aluno08 & 5.61 & 5.04 \\
\hline Aluno09 & 6.53 & 5.93 \\
\hline
\end{tabular}

\begin{tabular}{|l|l|l|}
\hline Meninas & $\begin{array}{l}1^{\circ} \text { teste } \\
\mathrm{T}(\mathrm{s})\end{array}$ & $\begin{array}{l}2^{\circ} \text { teste } \\
\mathrm{T}(\mathrm{s})\end{array}$ \\
\hline Aluno10 & 7.24 & 6.44 \\
\hline Aluno11 & 6.70 & 6.42 \\
\hline Aluno12 & 8.00 & 7.03 \\
\hline Aluno13 & 7.42 & 6.07 \\
\hline Aluno14 & 6.80 & 6.41 \\
\hline Aluno15 & 7.77 & 6.80 \\
\hline
\end{tabular}

Tabela 2: Resultado dos testes de Agilidade

Fica clara a evolução dos alunos de ambos os sexos comparando com os resultados anteriores. Muitos alunos do sexo masculino atingiram o desempenho muito bom ficando no tempo de 5,0 ou 5,2 segundos. A maioria das meninas que anteriormente estavam com o desempenho fraco ou razoável conseguiu atingir o desempenho bom ou muito bom.

Para Fortes e Mascarenhas (2010) a necessidade de avaliar a aptidão física fica evidente para podermos conhecer os alunos e saber a dificuldade deles ligada ao desenvolvimento, podendo assim atingir uma melhor performance na execução das atividades relacionadas com velocidade de deslocamento. Referindo-se ao teste de Velocidade de Deslocamento o desempenho maior dos meninos, pois durante o teste conforme mostra a Tabela 3, notasse que dos alunos do sexo masculino $55.5 \%$ atingiram um desempenho muito bom, $33.3 \%$ bom e $11.1 \%$ excelente. E no sexo feminino podemos perceber que $49.8 \%$ atingiram um desempenho bom, $16.6 \%$ muito bom e $16.6 \%$ razoável.

Comparando com o estudo de Verardi et al (2009) realizou este teste com 60 adolescentes de um projeto social, onde $94,1 \%$ do sexo masculino e 84,6 do sexo feminino 
tiveram o desempenho Fraco, razoável e muito fraco e o estudo de Dias, Podestá e Afonso (2007), que avaliaram 50 meninas onde 64\% tiveram o desempenho fraco, razoável e muito fraco. Fica evidente que os alunos da região Centro Oeste do Estado do RS estão com desenvolvimento acima dos participantes das pesquisas mencionas acima, e que é significativa à melhora do tempo comparando os resultados do $1^{\circ}$ e $2^{\circ}$ teste realizado.

Podemos observar a tabela com os resultados dos testes de velocidade de deslocamento que foram aplicados, demonstrando o desempenho do primeiro e segundo teste.

\begin{tabular}{|l|l|l|}
\hline Meninos & $\begin{array}{l}1^{\circ} \text { Teste } \\
\mathrm{T}(\mathrm{s})\end{array}$ & $\begin{array}{l}2^{\circ} \text { Teste } \\
\mathrm{T}(\mathrm{s})\end{array}$ \\
\hline Aluno01 & 3.62 & 3.15 \\
\hline Aluno02 & 3.48 & 3.00 \\
\hline Aluno03 & 3.36 & 3.20 \\
\hline Aluno04 & 3.38 & 2.70 \\
\hline Aluno05 & 3.22 & 3.01 \\
\hline Aluno06 & 3.45 & 3.03 \\
\hline Aluno07 & 3.40 & 3.05 \\
\hline Aluno08 & 3.34 & 3.02 \\
\hline Aluno09 & 3.41 & 3.22 \\
\hline
\end{tabular}

\begin{tabular}{|l|l|l|}
\hline Meninas & $\begin{array}{l}1^{\circ} \text { Teste } \\
\mathrm{T}(\mathrm{s})\end{array}$ & $\begin{array}{l}2^{\circ} \text { Teste } \\
\mathrm{T}(\mathrm{s})\end{array}$ \\
\hline Aluno10 & 4.02 & 3.81 \\
\hline Aluno11 & 3.50 & 3.05 \\
\hline Aluno12 & 4.20 & 3.80 \\
\hline Aluno13 & 4.54 & 4.21 \\
\hline Aluno14 & 4.05 & 3.77 \\
\hline Aluno15 & 3.80 & 3.65 \\
\hline
\end{tabular}

Tabela 3: Resultado dos testes de Velocidade de Deslocamento

Para que esse tema seja discutido podemos comparar a performance dos escolares na tabela acima com o estudo de Bergmann (2015) que do sexo masculino apenas 0,7\% atingiram o desempenho excelente, $13,2 \%$ muito bom e $25,3 \%$ bom, 19,4\% razoável e 41,5\% fraco. E do sexo feminino apenas 0,4\% atingiram o desempenho excelente, 11,8\% muito bom e 15,7\% bom, 19,6\% razoável e 52,5\% fraco. Isso demostra que os escolares avaliados estão com a aptidão física acima da pesquisa demonstrada por Bergmann et al (2015), Dias, Podestá e Afonso (2007) e Verardi et al (2009).

As evoluções dos alunos nos testes de Velocidade foram claras, fica evidente que os exercícios físicos demonstraram eficácia, outro teste que apresentaram bons resultados foi no teste de Resistência Geral. 
Segundo Machado (2012, p. 27) "a aptidão física relacionada à saúde cardiovascular compreende a composição corporal e a aptidão cardiorrespiratória e seus altos índices estão relacionados a riscos aumentados de doenças coronárias e síndrome metabólica". Sendo assim é fundamental saber o estado de saúde do individuo, para conseguirmos evitar doenças relacionadas à saúde cardiorrespiratória.

Este estudo mostrou evolução dos alunos do sexo masculino e feminino, os resultados dos testes de Resistência Geral conforme mostra a Tabela 4, apresentam que dos alunos do sexo masculino $33.3 \%$ atingiram o desempenho excelente $33.3 \%$ muito bom e $11.1 \%$ bom, $11.1 \%$ razoável e apenas $11.1 \%$ fraco. E no sexo feminino podemos perceber que $16.6 \%$ atingiram um desempenho excelente, $16.6 \%$ muito bom, $33.2 \%$ razoável e $33.2 \%$ fraco.

Alguns estudos realizados como, por exemplo, Guedes et al (2002) identificou dos adolescentes entre 15 e 18 anos que $56 \%$ das meninas e $76 \%$ dos meninos estavam com o desempenho fraco ou razoável. Ronque et al (2007) descreveu que $73 \%$ do sexo masculino e $68 \%$ do feminino estavam com baixo nível cardiorrespiratório. Após a análise dos estudos citados acima podemos perceber que a resistência geral dos alunos está baixa e com o estudo realizado durante as aulas de Educação Física com o objetivo de melhorar a aptidão física dos alunos demostra resultados positivos.

A tabela 4 com os resultados dos testes de Resistência Geral demostra a evolução dos participantes da pesquisa, demostrando os testes antes e depois da aplicação da UD.

\begin{tabular}{|l|l|l|}
\hline Meninos & $\begin{array}{l}1^{\circ} \text { Teste } \\
\mathrm{D}(\mathrm{m})\end{array}$ & $\begin{array}{l}2^{\circ} \text { Teste } \\
\mathrm{D}(\mathrm{m})\end{array}$ \\
\hline Aluno01 & 1423 & 1520 \\
\hline Aluno02 & 1100 & 1320 \\
\hline Aluno03 & 1126 & 1230 \\
\hline Aluno04 & 1153 & 1320 \\
\hline Aluno05 & 1508 & 1540 \\
\hline Aluno06 & 1540 & 1540 \\
\hline Aluno07 & 946 & 1100 \\
\hline Aluno08 & 983 & 1200 \\
\hline Aluno09 & 1220 & 1320 \\
\cline { 1 - 2 } & &
\end{tabular}

\begin{tabular}{|l|l|l|}
\hline Meninas & $\begin{array}{l}1^{\circ} \text { Teste } \\
\mathrm{D}(\mathrm{m})\end{array}$ & $\begin{array}{l}2^{\circ} \text { Teste } \\
\mathrm{D}(\mathrm{m})\end{array}$ \\
\hline Aluno10 & 866 & 980 \\
\hline Aluno11 & 1000 & 1080 \\
\hline Aluno12 & 780 & 860 \\
\hline Aluno13 & 880 & 990 \\
\hline Aluno14 & 912 & 1210 \\
\hline Aluno15 & 890 & 950 \\
\hline
\end{tabular}


Tabela 4: Resultado dos testes de Resistência Geral (6 min)

O teste de sentar e alcançar é um dos testes aplicados para estabelecer a flexibilidade. Segundo Machado (2012) a flexibilidade é dos fundamentais itens para a aptidão física, a avaliação da flexibilidade durante a adolescência é indispensável para a descoberta precoce de alteração na postura e para de precaução de futuros problemas.

Nos testes de Flexibilidade, verificando os resultados dos alunos do sexo masculino podemos perceber que $77 \%$ atingiram a zona de saúde e que $50 \%$ das alunas do sexo feminino atingiram a zona de saúde após a realização dos testes.

O domínio dos adolescentes na zona de risco a saúde foram superiores, principalmente no sexo masculino. Machado (2012, p. 88) diz que "Petroski et al (2012) encontrou 98,5\% dos adolescentes entre 14 e 17 anos na zona de risco no teste. Guedes et al (2002) em Londrina, estudou adolescente entre 15 e 18 anos, constando 60\% (ambos sexos) de inaptidão para força abdominal”. Comparando com estes testes já realizados fica evidente que a grande maioria dos adolescentes está na zona de risco. No teste de resistência abdominal verificando os resultados dos alunos do sexo masculino podemos perceber que apenas $22.2 \%$ atingiram a zona de saúde e $50 \%$ das alunas do sexo feminino atingiram a zona de saúde.

Além das questões culturais para Verardi et al (2009, p. 131) "uma maior experiência motora nos movimentos fundamentais atribuídos aos meninos, outros fatores podem ter refletido no resultado do teste, como fato de os meninos terem uma maior proporção de fibras de contração rápida", sendo assim podemos compreender que os meninos tem mais força explosiva que as meninas. No estudo de Verardi et al (2009) 61,7\% do sexo masculino e $34,6 \%$ do sexo feminino atingiram o desempenho "muito bom" ou "bom", comparando com os resultado dos testes de Força de Membro Superiores dos alunos da região Centro Oeste do RS verificando os alunos do sexo masculino podemos perceber que apenas $11.1 \%$ atingiram um desempenho muito bom, $11.1 \%$ bom, $33.3 \%$ razoável e $44.4 \%$ fraco. E no sexo feminino podemos perceber que $16.6 \%$ atingiram um desempenho bom, $33.2 \%$ razoável e $50 \%$ fraco.

Nos testes de Membros Inferiores comparando com os resultados de Verardi et al (2009) que realizou o teste com 60 adolescentes onde $47 \%$ estão com o índice "muito fraco" ou "fraco" e no estudo de Pereira et al (2012) que o desempenho "insatisfatório" e "fraco" chegou a $70 \%$ podemos observar que o desempenho dos alunos da região Centro Oeste do RS ficou bem acima dos apresentados por Verardi et al (2009) e Pereira et al (2012) pois nos testes de Força de Membro Inferiores verificando os alunos do sexo masculino podemos 
perceber que $33.3 \%$ atingiram um desempenho muito bom, 33.3\% bom, 22.2\% razoável e $11.1 \%$ fraco. E no sexo feminino podemos perceber que $33.2 \%$ atingiram um desempenho muito bom, $16.6 \%$ bom, $33.2 \%$ razoável e $16.6 \%$ fraco.

O presente estudo teve como objetivo verificar os efeitos que as aulas de Educação Física voltada pra o tema capacidades físicas podem ter em escolares, os alunos foram avaliando antes e depois da UD que teve como objetivo organizar, definir e especificar os conteúdos que foram trabalhados durante a pesquisa. A UD apresentou uma sequência de proposições que estavam de acordo com as necessidades e peculiaridades do grupo que foi trabalhado. Buscando conhecer as relações que se estabeleceram a partir do processo de ações, constituindo de forma significativa os visíveis efeitos do uso desse instrumento. Foi planejado pelo pesquisador o conteúdo agrupado com os objetivos conceituais, procedimentais e atitudinais, neste caso, a influência no desenvolvimento da aptidão física dos alunos.

Com os resultados obtidos fica evidente a influência das aulas, destaque para os efeitos nos testes de agilidade, velocidade de deslocamento, resistência geral e flexibilidade. Em geral a aptidão física dos participantes da pesquisa evoluiu bastante, comparando com outras regiões do Brasil, podemos compreender que os participantes da pesquisa estão com desempenho acima dos citados pelo texto.

\section{CONCLUSÃO}

O presente estudo com base nas amostras dos resultados percebe-se uma melhora significativa na aptidão física dos participantes da pesquisa. Ao analisar a aplicação de uma Unidade Didática coerente, fica evidente que há possíveis efeitos, já que, o desenvolvimento da aptidão física está em constante evolução e, possui uma gama de aspectos, no qual o corpo está em contínua ação e consequentemente adaptando-se conforme as intervenções recebidas.

Após a aplicação dos testes do PROESP (2015) realizados antes e após da UD com o tema capacidade física, com tempo de duração de três meses e comparando os dados coletados ficou evidente que os todos os objetivos foram alcançados e que as aulas tiveram efeitos positivos na aptidão física dos participantes da pesquisa. Sendo assim, entra em pauta a importância do papel do professor de Educação Física, este possui a oportunidade de ser influente e principalmente possuí a função de ensinar eficientemente, pois o mesmo se encontra em uma posição favorecida para criar instrumentos que aumentam a aptidão física 
dos alunos. Sugerem-se mais estudos de natureza longitudinal, pois assim saberemos melhor a ação das aulas no desempenho.

Podemos concluir que a maioria dos alunos que participaram da pesquisa encontra-se na zona de saúde conforme os padrões estabelecidos pelo PROESP (2015). Os maiores percentuais dos alunos nos testes realizados depois das intervenções foram: Testes de Flexibilidade, Agilidade, Velocidade de Deslocamento e Resistência Geral.

\section{REFERÊNCIAS}

BERGMANN, Gabriel Gustavo; MELLO, Júlio Brugnara; HERNANDEZ, Mariele da Silva; FARIAS, Vinicius Martins; PINHEIRO, Eraldo dos Santos. Aptidão física relacionada ao desempenho motor de adolescentes de Uruguaiana, Rio Grande do Sul. Rev. bras. Ci. e Mov 2015;23(4): 72-79. 2015.

BORGES, Robson Machado. Diálogos sobre o ensino do esporte educacional: uma pesquisa-ação na formação continuada. Universidade Federal do Rio Grande do Sul Programa de Pós-Graduação em Ciências do Movimento Humano. Porto Alegre, BR-RS 2014. Disponível em: <http://hdl.handle.net/10183/101487>. Acesso em: 23 de Agosto de 2016.

CORSEUIL, Maruí Weber; PETROSKI, Edio Luiz. Baixos níveis de aptidão física relacionada à saúde em universitários. Revista Brasileira de Educação Física e Esporte, São Paulo, v.24, n.1, p.49-54, jan./mar. 2010.

DIAS, D. F.; PODESTÁ, R. C.; AFONSO, C. A. Como identificar um possível talento. Revista da Educação Física / UEM. Maringá, v. 18, supl., p. 422 a 425, 2007.

FARIAS, Edson dos Santos; CARVALHO, Wellington Roberto Gonçalves de; Ezequiel Moreira GONÇALVES; JÚNIOR ,Gil Guerra. Efeito da atividade física programada sobre a aptidão física em escolares adolescentes. Rev Bras Cineantropom Desempenho Hum , 12(2):98-105, 2010.

FORTES, Antonio Junior Lopes; MASCARENHAS, Josiene de Lime. A prática de atividade física: promovendo a saúde - UNINORTE 2010.

GAYA, Adroaldo Cezar Araújo. Projeto esporte Brasil: manual de testes e avaliação/Adroaldo Gaya, Anelise Gaya. Porto Alegre: UFRGS, 2015.

GIDDENS, Anthony. Sociologia. Tradução: Sandra Regina Netz. 6 ed. Porto Alegre: Artmed, 2012.

GIL, Antônio Carlos. Métodos e técnicas de pesquisa social. 6. ed. São Paulo: Atlas, 2008.

GONZÁLEZ, Fernando Jaime; FENSTERSEIFER, Paulo Evaldo (Orgs.). Dicionário Crítico de Educação Física. 3 ed. rev. e amp. Ijuí/RS: UNIJUI, 2014. 
GUEDES, Dartagnan Pinto; ELISABETE, Joana; GUEDES, Ribeiro Pinto; SABATINI, Decio; OLIVEIRA, Jair Aparecido de. Atividade física habitual e aptidão física relacionada à saúde em adolescentes. Revista Brasileira de Ciência e Movimento, v. 10, n.1, p. 13-21, 2002.

MACHADO, Débora Teixeira. Perfil da aptidão física relacionada à saúde de escolares brasileiros avaliados pelo projeto Esporte Brasil: um estudo de tendência de 2003 a 2011. 100 f. Dissertação (mestrado). Escola de Educação Física, Programa de Pós-Graduação em Ciências do Movimento Humano, Universidade Federal do Rio Grande do Sul, Porto Alegre, 2012.

PEREIRA, Cleilton Holanda; FERREIRA, Debora da Silva; COPETTI, Giano Luis; GUIMARÃES, Leandro Corrêa; BARBACENA, Marcella Manfrin; LIGERI Natalia ;LOBATO, Oséias Guimarães Castro Silvia; DAVID, Ana Cristina de. Aptidão física em escolares de uma unidade de ensino da rede pública de Brasília-DF. Revista Brasileira Atividade Física Saúde. 2012;16:223-227.

PETROSKI, Edio Luiz; OLIVEIRA, Gustavo de; SILVA, Diego Augusto Santos; MAGGI, Rosana Mengue; FARIAS, Joni Marcio de. Fatores sociodemográficos e de aptidão física associados a baixos níveis de atividade física em adolescentes de uma cidade do sul do Brasil. Revista de Educação Física/UEM, v. 23, n. 4, p. 635-645, 4. trim. 2012.

RONQUE, Enio Ricardo Vaz; CYRINO, Edilson Serpeloni; DÓREA, Valfred; SERASSUELO JÚNIOR, Helio; GALDI, Enori Helena Gemente; ARRUDA, Miguel de. Diagnóstico da aptidão física em escolares de alto nível socioeconômico. Rev Bras Med Esporte vol.13 no.2 Niterói Mar./Apr. 2007.

VERARD, Carlos Eduardo Lopes; Da SILVA, Ana Paula Lobo; DO AMARAL, Vilton Eder; DE LIMA, Vinicius Freitas; HIROTA, Vinicius Barroso. Análise da aptidão física relacionada à saúde e ao desempenho motor em crianças e adolescentes da cidade de Carneirinho-MG. Mackenzie Rev Educ Phys Esporte. 2009; 6:127-134. 